\title{
INFORME DEL RELATOR
}

\author{
Bernal Herrera \\ Universidad de Costa Rica
}

La tarea de relator en un seminario conlleva aspectos gratos e ingratos conocidos de todos los que alguna vez hayan cumplido dicho rol. En lo grato, posibilita una activa participación sin la responsabilidad implícita en la presentación de una ponencia propia; en lo ingrato, impone la tarea de armar un relato que rinda cuenta de lo sucedido basado en exposiciones ajenas y en discusiones que a menudo se disparan en muy opuestas direcciones. Se trata, en suma, de extraer una cierta coherencia de una masa de material a menudo muy dispar, evitando por igual tanto la imposición de marcos unitarios tal vez inexistentes fuera de la mente del relator, como la mera reproducción del vaivén temático y argumentativo de las ponencias individuales y sus respectivas discusiones. Intentaré en lo que sigue dar una visión que vaya más allá tanto de lo puramente impresionista como del análisis individual de las ponencias que integraron las sesiones cuya moderación tuve a mi cargo. Para ello he centrado el análisis alrededor de tres áreas temáticas que, según mi opinión, han dominado tanto las presentaciones como las discusiones.

\section{Las taxonomías}

El primer eje temático, y uno de los que considero más significativos por su importancia para la actividad crítica en general, es el de las taxonomías literarias. Por momentos se intentó reducir o incluso negar el problema, considerando que la argumentación se estaba dando alrededor de "etiquetas", pero la constante resurgencia del tema obligó a quienes tal hicieron a reconocer la imposibilidad de, valga la paradoja, etiquetar el problema como uno de meras etiquetas.

La primera manifestación de este primer eje se dio en el arranque mismo del seminario con la ponencia de Blanco Aguinaga, quien al abordar el problema del significado y sentido de los términos modernidad y modernismo, llamó la atención a las dificultades de conversión terminológica entre diferentes esferas culturales, observación plenamente sostenible. Bástenos recordar que tanto la modernidad como el modernismo literario, fenómenos cuyas relaciones son exploradas por el texto de Blanco, significan muy diversas cosas en diversos ámbitos culturales. En su exploración del posible imperialismo cultural subyacente en la aplicación del concepto de "modernidad" propio de la cultura anglosajona al ámbito hispánico, Blanco hizo ver que una importación sin más de dicho concepto puede acabar marginando la esfera cultural hispánica 
del proceso mismo de modernización. Con ello afloró uno de los niveles de discusión del problema: el de hacer ver que toda taxonomía, más que un vacuo juego de etiquetas superfluas o intercambiables, implica un esquema de interpretación de la realidad con ella clasificada, esquema que necesariamente ayuda a determinar la concepción, y por ende la valoración, que tengamos de dicha realidad.

El segundo nivel de discusión del problema de las taxonomías se centró en sus manifestaciones más estrictamente literarias, tema también presente en la ponencia de Blanco en su alusión a los divergentes significados que el término 'modernismo' posee en las historias literarias hispanas y anglosajona y, añado yo, en las literaturas de lengua portuguesa. Recordemos, en efecto, que lo que la historiografía literaria anglosajona y luso-brasileña llama modernismo no coincide con el modernismo hispano(americano), sino más bien con lo que la historiografía de esta última literatura denomina las vanguardias. Este tema de las taxonomías literarias se mantuvo a lo largo del seminario, reapareciendo diversa pero constantemente en las ponencias de Anthony Zahareas, Ivonne Robles y Jorge Chen. La de Robles llamó la atención a las dificultades criticas que surgen durante el intento de clasificar un texto como El jaúl, de Max Jiménez, cuya exclusión del canon oficial de la literatura costarricense podría deberse, entre otras cosas, a esta dificultad. Robles revisa sucintamente la bibliografía sobre El jaúl, siendo sorprendente que el texto haya sido clasificado, por ejemplo, como texto indigenista, teniendo en cuenta que si bien es cierto el narrador menciona los indígenas, dicha mención es motivada por la completa ausencia de éstos de la espacio-temporalidad definida por la obra. La clasificación de indigenista de un libro ninguno de cuyos personajes es indígena ilustra de manera patente el problema de las nomenclaturas literarias. Robles plantea como posible vía de ataque al texto su estudio a la luz tanto de la corriente neobarroca, tal y como ésta es definida por Severo Sarduy, como de la estética esperpéntica de Valle-Inclán. Ciertamente la utilización del concepto de 'neobarroco' parece más prometedora que la de la categoría 'indigenista', en especial por la gravitación de lo grotesco, concepto presente en la definición de Sarduy y ciertamente actuante en la novela de Jiménez. Considero, sin embargo, que la superficie estilística de El jaúl, texto más bien descarnado y sucinto, no parece responder adecuadamente a la definición de Sarduy de neobarroco, término con que alude a una escritura altamente proliferante y metafórica. Bastante más fecunda pereciera la segunda opción presentada por Robles, la aplicación del concepto valleinclaniano de esperpento como posible categoría de estudio de la novela de Jiménez.

La ponencia de Zahareas, centrada en el problema de los géneros en Valle-Inclán, también generó discusiones directamente relacionadas con la taxonomía literaria, ya que el intento de equiparar la variadísima nomenclatura utilizada por Valle-Inclán en sus numerosos textos con una clasificación genérica, pareciera tener como consecuencia la fundación de géneros completamente nuevos. Hubo cierto acuerdo en que tal equiparación no podía sostenerse, pasando la discusión a otro punto de la ponencia de Zahareas, el de la transformación sufrida por la tragedia clásica en manos de Valle-Inclán. Una vez más, se vió la dificultad de clasificar los textos de Valle-Inclán apelando a géneros cuyo rompimiento es, casualmente, uno de los objetivos de su autor. En cuanto a la ponencia de Jorge Chen, ejemplificó las dificultades que surgen en la lectura de un texto como La lámpara maravillosa en virtud de sus diferentes ubicaciones genéricas. La ponencia hizo reflexionar sobre las muy diversas expectativas generadas por La lámpara maravillosa de acuerdo a si se lee el texto como un ensayo estético, una autobiografía, una novela o un tratado ocultista. 
Las anteriores discusiones hacen ver cómo, en crítica literaria, las taxonomías son mucho más que juegos intercambiables de etiquetas, por determinar no sólo la ubicación de los diversos textos en escuelas, géneros, estilos, etc., sino también en buena medida las expectativas de lectura, y en esa medida el juicio que sobre los textos se haga. Dada la imposibilidad de ejecutar lecturas totalmente ingenuas, la clasificación de un texto bajo una u otra de la rúbricas disponibles determina parcialmente su sentido. Así, no es lo mismo leer las obras de Valle-Inclán como adaptación, parodia o reformulación del clásico género de la tragicomedia, que leerlas como fundadoras de un nuevo género: el esperpento. La discusión, claro está, no se limita al problema de la adopción de un nombre u otro ni al de las diferentes expectativas generadas por las diversas nomenclaturas, sino al sentido mismo de éstas. En el caso concreto de Valle-Inclán, ¿es su esperpento un nuevo género o una nueva estética? La clasificación misma de su obra dentro de algún movimiento literario dista de ser clara. ¿Es un modernista o un precursor del vanguardismo? ¿Acaso un autor que tras empezar como modernista acabó como vanguardista? Algunos críticos han insinuado que su singularidad escritural es tal que imposibilita toda clasificación de su obra. ¿Es ello cierto, y de ser así cuáles son sus relaciones con el modernismo y la vanguardia? He aquí el tipo de preguntas planteadas pero no siempre respondidas durante el seminario en lo que se refiere al tema de las taxonomías.

\section{Modernismo y modernidad}

El tema de las relaciones entre el modernismo y la modernidad está ciertamente emparentado con el de las taxonomías literarias expuesto en el aparte anterior, pero tanto su importancia como su constante presencia en ponencias y discusiones durante el seminario lo convierten en el segundo eje de exposición. Recordemos, por lo demás, que el seminario se convocó para discutir el tema de las relaciones entre Valle-Inclán y la modernidad.

Desde un principio se vió que la discusión de las relaciones entre el modernismo en tanto práctica escritural, y la modernidad en tanto proceso socio-histórico no podía, sin ser forzado, ser circunscrito al problema de las nomenclaturas literarias, por tocar de lleno otro problema central de la crítica literaria: el de las relaciones entre los textos y el contexto social. La discusión al respecto se vió enriquecida por la presencia de críticos que, convocados no por afinidades teóricas sino por un núcleo temático, representaban muy diversas orientaciones críticas, y por ende muy diversos modos de concebir las relaciones entre los textos y sus contextos. Desde una visión como la de Rodolfo Cardona, quien en su ponencia sobre la deconstrucción de la historia en Martes de Carnaval nos hizo ver, muy detalladamente, la reelaboración histórica efectuada en el texto de Valle-Inclán de hechos directamente tomados de su contexto socio-histórico, hasta ponencias como la de Robles, concentradas en aspectos más estrictamente escriturales y menos relacionados con el contexto social de la escritura, diversas posiciones sobre las relaciones textolcontexto fueron expuestas y discutidas. En parte como resultado de la heterogeneidad de los participantes, no se llegó a acuerdos finales respecto a tales relaciones, pero la discusión probó ser altamente productiva para sus participantes.

Como muchas de las discusiones, la concerniente a las relaciones entre el modernismo y la modernidad se inició con la ponencia de Blanco Aguinaga, quien planteó el problema de la autonomía usualmente implícito en las principales conceptualizaciones de la escritura moder- 
nista. En un principio el debate se centró en el complejo problema de los modos de representación y reformulación de la realidad socio-histórica presentes en los textos modernistas en general y de Valle-Inclán en particular. Si durante las discusiones sobre las taxonomías literarias hubo relativo acuerdo en que los modos asumidos por tal representación es muy diferente dependiendo de si nos referimos al modernismo hispano, al 'modernism' anglosajón o al 'modernismo' lusobrasileño, propietarios estos últimos de modalidades de representación más afines a lo que la nomenclatura hispánica denomina las vanguardias, la variedad de modos de representación y reformulación textual de la realidad externa se traslada aquí al interior del modernismo hispánico. Recordemos que la imagen tradicional de dicho movimiento, en especial la representada por figuras como la de Rubén Darío, nos suele hacer pensar, con justicia o sin ella, en una literatura que se quiere autónoma del contexto socio-histórico que marca su surgimiento. El modernismo, se ha afirmado numerosas veces, es una literatura torremarfilista, exotista o incluso abiertamente escapista. En lo referente a esta tema, la pluralidad de puntos de vista expuestos y discutidos en el seminario no impidió la aparición de un refrescante consenso general sobre la complejidad de las relaciones entre las realidades externa y textual al interior de la escritura modernista, consenso que evidencia el largo camino que han recorrido los estudios dedicados al modernismo, los que parecen haber superado de una vez por todas la simplista imagen arriba apuntada del modernismo como una literatura meramente escapista, imagen prevaleciente hasta no hace muchos años. Hubo consenso, entonces, en que los textos modernistas establecen relaciones altamente complejas con su realidad socio-histórica, no reducibles al tradicional torremarfilismo adscrito al movimiento según su imagen tradicional, pero tampoco, claro está, a mimetismo alguno de los practicados por la escritura realista. Esta complejidad aparece de manera patente en los textos modernistas de Valle-Inclán, si es que nos decidimos a clasificarlos en tal escuela pese a sus numerosos rasgos específicos. Los textos de Valle-Inclán, en efecto, presentan una curiosa mezcla de esteticismo, estilización lingüística e intencionalidad política, rasgos cuya diversa priorización lo mismo permiten distanciarlo del modernismo para acercarlo, por un lado, a la literatura de tesis, y por el otro a la escritura vanguardista, como efectuar la maniobra inversa, dependiendo de a qué factores escriturales decida el crítico prestarie más atención en su lectura.

El problema de las relaciones entre los textos modernistas y su contexto socio-histórico se desdobla de acuerdo con los factores que prioricemos en su discusión. El primer aspecto es el ya mencionado de los modos de representación y reformulación de la realidad socio-histórica al interior de dichos textos. Aquí la pregunta sería: ¿qué tipo de trabajo con los elementos tomados del contexto histórico efectúa la escritura modernista, y cuál es la importancia real de tales elementos en dicha escritura? Problemas como el de la debatida autonomía escritural del modernismo, presente en la ponencia de Blanco, el de la imagen del campesino costarricense en El jaúl de Max Jiménez expuesto por Robles, o el juego entre la representación y la construcción del sujeto autobiográfico en La lámpara maravillosa analizado por Chen, son todos aspectos que pertenecen a este nivel de discusión, nivel explícitamente tratado por Zahareas en su exposición al referirse a las relaciones entre el esteticismo y la política en los textos de Valle-Inclán. En este primer nivel no hubo un acuerdo sobre las respuestas específicas a la pregunta arriba planteada, pero sí lo hubo, ya lo dijimos, sobre la extrema complejidad de dichas relaciones. Con lo cual llegamos al segundo nivel de discusión: el de la importancia de los contextos sociohistóricos ya no al momento de la escritura de los textos modernistas, sino de su lectura. 
La discusión de este aspecto del problema evidenció una previsible correlación entre las estrategias asignadas a la escritura modernista y las modalidades de lectura aplicadas en su análisis. Así, las lecturas de quienes priorizan el contexto sociohistórico como factor determinante en la escritura, suelen encontrar en los textos de Valle-Inclán numerosos ejemplos de utilización y reformulación de elementos históricos, lo que ciertamente ayuda a romper la imagen tradicional del modernismo como una escritura puramente preciosista y autonómica. Quien esto escribe, por ejemplo, se vió obligado a recordar la importancia de conocer dichos contextos oyendo ponencias como las de Cardona, cuya meticulosa información histórica arrojó nueva luz sobre la obra Martes de carnaval. A su vez, quienes tienden a priorizar los aspectos intra e intertextuales hicieron ver el grado de autonomía presente en la escritura modernista, así como la imposibilidad de sujetar su análisis a los procesos referenciales en ella contenidos y referentes a su contexto histórico.

El tercer y último nivel en la discusión de las relaciones entre el modernismo y la modernidad es el que más se ciñe a su formulación, y tiene que ver principalmente con las mutuas adscripciones y territorialidades de tales términos. Aquí reaparece, una vez más, el problema de las etiquetas, ya que resulta imposible decidir la adscripción o no del modernismo al proceso histórico de la modernidad si no se definen de antemano ambos términos. Las discusiones al respecto arrojaron un desacuerdo que delineó, básicamente, dos posiciones. La primera es la de quienes colocan el modernismo literario en el interior del más amplio fenómeno de la modernidad haciendo del primero una manifestación literaria del segundo, caso de Blanco Aguinaga y, en menor escala, de Zahareas; la segunda la de quienes preferirían deslindar claramente ambos términos, caso de Peggy von Mayer.

Quien esto escribe considera que la dificultad de lograr un acuerdo sobre la importancia de los factores sociohistóricos en el estudio del modernismo, y de la literatura en general, refleja la disyuntiva básica que tal decisión implica a la hora de efectuar estudios literarios. Las diferentes respuestas dadas a esta disyuntiva marcan de algún modo los estudios literarios y la evolución de las escuelas críticas, las cuales presentan una gama de soluciones que va de la meticulosidad sociohistórica pregonada por el positivismo hasta el casi total abandono de tales factores en escuelas como el formalismo y la deconstrucción, pasando por la solución intermedia representada por la sociocrítica, una de las escuelas más influyentes en la critica literaria costarricense contemporánea.

Quisiera acabar lo relativo a este segundo eje temático ejemplificando las implicaciones que las decisiones concernientes a estos problemas y disyuntivas tienen en los estudios literarios en general, usando como caso concreto el modernismo. Siendo obvia la importancia de las decisiones tomadas en los dos primeros niveles, o sea en lo referente a las relaciones entre el texto y el contexto tanto al momento de su escritura (primer nivel) como de su lectura (segundo nivel), ejemplificaré algunas de las posibles implicaciones anexas al tercer nivel, el de las relaciones entre el modernismo y la modernidad, en apariencia el nivel más inocuo de todos. Adscribir o no el modernismo literario al fenómeno de la modernidad conlleva consecuencias serias en su estudio. Así, hacer del modernismo parte integrante de la modernidad podría llevarnos, por ejemplo, a la necesidad de enfatizar la marginalidad y dependencia de la muy incompleta modernidad hispana y latinoamericana, lo que a su vez induciría a asumir y enfatizar el carácter periférico general de los movimientos literarios hispanoamericanos, incluido el modernismo. 
Ello por cuanto la inserción de un movimiento literario, aun de uno tan desarrollado como el modernismo, en el seno de un fenómeno tan incompleto como el de la modernidad latinoamericana de fines de siglo, necesariamente deberá referirnos al problema de la marginalidad. Independizar, por otro lado, el modernismo de la modernidad, enfatizando en su estudio no su inserción en un proceso sociohistórico sino en la especificidad del desarrollo literario, permite ponerlo en igualdad de condiciones con los movimientos literarios coetáneos, eliminándose así toda marginalidad estrictamente literaria. Aunque parece la más recomendable, esta última posición tampoco carece de riesgos, ya que podría llevar a ignorar la problemática del contexto social en que surge el movimiento. Tal precio, sin embargo, parece menor que el extraído por la primera posición.

\section{Centro y periferia}

El mencionado problema de la marginalidad o no de la literatura modernista en lengua española dependiendo de su adscripción o no al más amplio fenómeno de la modernidad, nos lleva directamente al tercer eje de las discusiones sostenidas durante el seminario, el referente al centro y la periferia. Este tema, como los otros dos, empezó a discutirse a partir de la ponencia de Blanco Aguinaga, y si bien no mantuvo la centralidad que al principio parecía iba a tener, se siguió discutiendo hasta el final del seminario. Blanco Aguinaga se refiere en su ponencia a los problemas asociados con el imperialismo, el colonialismo y el neocolonialismo culturales, temas ampliamente discutidos en la crítica literaria contemporánea y que giran, evidentemente, alrededor de los conceptos de centro y periferia. Sin plantear soluciones al respecto, la ponencia de Blanco recordó desde un principio a todos los participantes nuestra adscripción a una esfera cultural usualmente considerada marginal, así como las implicaciones que tal situación acarrea en los estudios literarios de movimientos generados en órbitas marginales, tales como el modernismo hispano(americano). Este fenómeno es evidente en el caso de Valle-Inclán, cuya marginalidad en las historias oficiales de la literatura occidental contrasta abiertamente con su centralidad en las literaturas de lengua española.

Los procesos de marginalización discutidos no sólo ocurren en la interacción entre culturas diversas, sino también al interior de culturas particulares, aún de aquellas tan espacio-temporalmente reducidas como la costarricense. En este nivel lo que tenemos es el muy discutido problema del canon literario, punto tocado por Robles al analizar la posición de escritores como Max Jiménez, marginado del canon de la literatura costarricense por motivos no completamente claros. Problemas similares de constitución y recomposición del canon también se discutieron respecto a las obras de Valle-Inclán. Así, César Oliva afirmó en reiteradas oportunidades la insostenibilidad de la imagen de Valle-Inclán como un autor marginado o censurado, refiriéndose a la constante, aunque poco exitosa hasta muy recientemente, presencia de sus dramas en las tablas españolas. Víctor Valembois cerró la sesión de ponencias del seminario refiriéndose a las dificultades experimentadas por Valle en razón de lo avanzado de su teatro, en especial del tardío. Esta división de Valembois trasladó la discusión sobre el centro y la periferia de los cánones nacionales al interior de la obra de los autores individuales, en este caso de Valle-Inclán. A este respecto la discusión se centró en si realmente se justifica el corte hecho por numerosos críticos entre el Valle-Inclán modernista y el esperpéntico, y más aún si habría que discriminar 
al primero, ello es, si se justifica hacer del hipotético primer Valle-Inclán un autor periférico opuesto al Valle-Inclán central, el del esperpento. Con esta discusión, que generó un cierto acuerdo sobre la inconveniencia de establecer una separación radical al interior de la producción de Valle-Inclán que diferenciaría uno central y otro periférico, se cerraron las sesiones de ponencias del seminario. Después vendría la presentación de los relatos, a cargo de María Amoretti y de un servidor, los que en general tuvieron buena acogida y fueron poco discutidos.

Terminaré con unas pocas palabras sobre un aspecto organizativo que influyó grandemente en su nivel intelectual. La organización del seminario, pocos ponentes y textos largos, permitieron análisis más sostenidos que los usuales en este tipo de actividades, pudiendo decirse que hubo menor saturación temática y mayor profundidad crítica. El reducido número de participantes también produjo una más constante e intensa interacción, lo que permitió darle mayor continuidad al diálogo. La concentración temática, a su vez, impuso un giro interesante a la discusión, ya que al girar el seminario alrededor de núcleos temáticos y no teóricos, hubo ponentes de muy diversas corrientes críticas, lo que enriqueció la discusión debido a las amplias diferencias conceptuales en el tratamiento de los temas. Constantemente se dieron discusiones ya no sobre problemas concretos, sino sobre la definición misma de cuáles problemas valía la pena discutir. Considero que el seminario fue todo un éxito, y deseo hacer constar por escrito la gratitud de sus participantes con las instituciones y organismos patrocinadores, y muy especialmente con Clara Ballesteros y el Centro Cultural Español por su magnífica hospitalidad. 
